\title{
DER MYTHOS VON DEN HUNG-KƠNIGEN UND DAS NATIONALE SELBSTVERSTÄNDNIS DER VIETNAMESEN
}

\author{
Von NGUYen Thanh Hung
}

Die vorliegende Untersuchung befaßt sich mit der neueren Interpretation der Vor- und Frühgeschichte Vietnams durch die Historiker der Sozialistischen Republik Vietnam. Es geht uns dabei jedoch weniger um ein historisches Interesse, sondern vielmehr darum, anhand dieser Deutung aufzuzeigen, daß sich die herrschende Ideologie in Vietnam keineswegs im Lehrgebäude des Marxismus-Leninismus erschöpft, wie gemeinhin angenommen wird. Wie in allen Ländern der Dritten Welt steht auch in Vietnam die Frage nach der nationalen Identität im Prozeß der Dekolonisation im Vordergrund. Der Nationalismus in der Dritten Welt nährt sich so zum entscheidenden Teil von einer Ideologie, in der die eigene Vergangenheit und Tradition verherrlicht und romantisch verklärt werden. Hierin macht der vietnamesische Nationalismus auch keine Ausnahme. Dieser Tatbestand, der sonst in der offiziellen Ideologie verhüllt bleibt, soll in der folgenden Darstellung der Interpretation der Vor- und Frühgeschichte in Vietnam verdeutlicht werden.

In einem 1941 verfaßten, von China aus an die Landsleute gerichteten Briefe, in dem sie zum Aufstand gegen die Franzosen und Japaner aufgerufen wurden, beschwor Hô Chi Minh auf eindringliche Weise, daß ,,mehr als zwanzig Millionen Abkömmlinge vom Geschlecht Lac-Hông nunmehr entschlossen sind, nicht länger das Schicksal der Sklaven zu erdulden “1. Später prägte er während des Indochina-Krieges das folgende, inzwischen in Vietnam populär gewordene Wort: ,,Die Hùng-Könige haben das Verdienst gehabt, unseren Staat zu gründen, und nun ist uns als ihren Nachkommen die Pflicht aufgegeben, ihn gemeinsam zu verteidigen“" (Các vua Hùng da có công dung nuóc, Bác cháu ta phai cùng nhau giu lây nuóc). In beiden Fällen nahm Hô Chi Minh Bezug auf die Úberlieferung von den Hùng-Königen, der zufolge einst im Kerngebiet des alten vietnamesischen Staates (Tonkin und Nord-Annam) vor der Zeit der ersten chinesischen Eroberung (im dritten Jahrhundert v. u. Z.) ein Königreich namens Van-lang existierte, dessen Gründer die Hùng-Könige sein sollten. Die Hùng-Könige gehörten danach zum Geschlecht Lac-Hông, das aus der Vereinigung der Geschlechter des Drachens (rông) und der Fee (tiên) hervorgegangen sein sollte. Uber achtzehn Generationen sollten sie das Reich Van-lang regiert haben, bis es im dritten Jahrhundert v. u. Z. den Eroberern aus dem Norden zum Opfer fiel ${ }^{2}$. Die Anspielung von Hô Chi Minh auf diese mythischen Gestalten und ihre heroischen Taten hat in diesem Kontext eine kaum zu verkennende politische Implikation: Die Vietnamesen, die im Laufe ihrer Geschichte fast ununterbrochen einer Fremdherrschaft ausgesetzt waren, sollten an ihre nationale Identität erinnert werden, und zugleich sollte der Legitimität des Kampfes um die Wiedergeburt der vietnamesischen Nation dadurch Nachdruck verliehen werden.

Der Mythos von den Hùng-Königen wurde zum ersten Mal in den Kompilationen ,,Viêt diên u linh tâp“ (Erzählungen über die unsichtbaren Mächte des Landes Viêt) von Lý Tê Xuyên und ,Linh-nam chích quái“ (Erzählungen über die außergewöhnlichen Wesen von Linh-nam) von Trân Thê Pháp niedergeschrieben, und zwar erst im 14. bzw. 15. Jahrhun-

\footnotetext{
* Es ist für den Verfasser eine angenehme Pflicht, an dieser Stelle auf die Unterstützung des Volkswagenwerks hinzuweisen, ohne die die vorliegende Untersuchung nicht möglich gewesen wäre.

1 Hô Chí Minh, De la révolution 1920-1966; herausgegeben von Bernard Fall, Paris 1968, S. 180.

2 Vgl. Lê Thanh Khoi, 3000 Jahre Vietnam, München 1969, S. 19 f.
} 
$\operatorname{dert}^{3}$, also zu einer Zeit, da das vietnamesische Kaiserreich vor seiner Glanzzeit stand ${ }^{4}$. Die Intention der Autoren ist klar: Die Glorifizierung der Vergangenheit sollte offensichtlich den nationalen Selbstbehauptungswillen der Vietnamesen gegenüber dem übermächtigen China stärken. Deshalb schrieben sie dem Reich Van-lang eine beträchtliche Ausdehnung zu: Es sollte sich nach Norden bis zum Tung-t'ing-See (im Süden des heutigen China) und nach Süden bis zur Grenze des einstigen Königreichs Champa ${ }^{5}$ erstrecken. Die Gründung von Van-lang wurde von den früheren Historiographen sogar präzise auf 2879 v. u. Z. datiert $^{6}$. Der Vorfahre der Hùng-Könige, nämlich Kinh Duong vuong, zeichnete sich dieser Uberlieferung zufolge besonders durch seine Heiligkeit und Weisheit aus und war somit in jeder Hinsicht seinem älteren Bruder, dem legendären chinesischen Herrscher Ti-I (vietnamesisch: Dê-Nghi) ebenbürtig, übertraf diesen jedoch in seiner Bescheidenheit, einer Eigenschaft, die in der Wertvorstellung der Vietnamesen hochgeschätzt wird ${ }^{7}$.

Bald nach der schriftlichen Fixierung wurde der Mythos von den Hùng-Königen in die offiziellen Geschichtswerke der verschiedenen vietnamesischen Dynastien als Darstellung der Entstehung des vietnamesischen Staates und des Ursprungs des vietnamesischen Volkes aufgenommen, obwohl die früheren Historiographen sich durchaus bewußt waren, daß der Gehalt dieser Uberlieferung mit keinen historischen Gegebenheiten in Verbindung gebracht werden konnte ${ }^{\mathbf{8}}$. Dabei ist es jedoch interessant festzustellen, daß bei ihrer Wiedergabe im Laufe der Zeit Anderungen inhaltlicher Details immer wieder vorgenommen wurden. Vergleicht man die Aufzeichnung im Geschichtswerk ,Dai Viêt su ký toàn thu“ (Vollständige Annalen von Groß-Vietnam), das von Ngô Si Liên im 15. Jahrhundert verfaßt wurde, mit derjenigen in ,,Khâm dinh Viêt su thông giám cuong muc“ (Auf kaiserlichen Befehl verfaßte Texte und Kommentare des allgemeinen Spiegels der Geschichte von Vietnam), das die Hofhistoriographen der Nguyên-Dynastie im 19. Jahrhundert kompilierten, so fällt auf, daß der Umfang der Uberlieferung durch Ergänzung verschiedener aus chinesischen Quellen stammender Einzelheiten zunahm und vor allem mythische Elemente zum Teil herausgenommen wurden ${ }^{9}$. Dabei werden die Bemühungen der früheren Historiographen deutlich: Sie wollten offensichtlich durch diese Rationalisierung den Mythos von den Hùng-Königen glaubhafter erscheinen lassen.

In den ersten Jahrzehnten dieses Jahrhunderts wurde das Reich Van-lang der Hùng-Könige von den französischen Gelehrten H. Maspéro und L. Aurousseau zum Gegenstand ihrer Forschung gemacht. Durch eine gründliche Auswertung vorhandener chinesischer Quellen kamen sie zu dem Ergebnis, daß es die Hùng-Könige nicht gegeben haben könne ${ }^{\mathbf{1 0}}$. Die Vorfahren der Vietnamesen sollten vielmehr aus dem Reich der Yue (vietnamesisch: Viêt) im Gebiet des unteren Jangtse-kiang in China stammen. Von den nördlichen Völkergruppen

3 Vgl. E. Gaspardone, Bibliographie annamite, in: Bulletin de l'Ecole Française d'Extrême-Orient (BEFEO), XXXIV, 1935.

4 Das 15. Jahrhundert unter der Lê-Dynastie gilt als das , ,goldene Zeitalter" der vietnamesischen Geschichte; s. Lê Thanh Khoi, a. a. O., S. $155 \mathrm{ff}$.

5 D. h. bis zu dem sogenannten Wolkenpaß, der im 14. Jahrhundert den südlichsten Punkt des vietnamesischen Kaiserreichs darstellte, heute jedoch fast genau in der Mitte des Territoriums Vietnams liegt.

6 Ngô Si Liên, Dai Viêt su ký toàn thu (Vollständige Annalen von Groß-Vietnam), Bd. 1, Hanoi 1967, S. 63.

$7 \mathrm{Vgl}$. Yamamoto Tatsuro, Myths Explaining the Vicissitudes of Political Power in Ancient Vietnam, in: Acta Asiatica, Nr. 18, Tokyo 1970, S. 82.

8 NgôSi Liên, a. a. O., S. 69 f.; auch ,,Khâm dinh Viêt su thônggiámcuongmuc“ (Auf kaiserlichen Befehl verfaßte Texte und Kommentare des allgemeinen Spiegels der Geschichte von Vietnam), Bd. 1, Saigon 1965, S. 8b

9 Vgl. Yamamoto Tatsuro, a. a. O., S. 80.

$10 \mathrm{H}$. Maspéro konnte in seinen gründlichen Untersuchungen der chinesischen Quellen, auf denen die Darstellung über die Hùng-Könige der vietnamesischen Historiographen hauptsächlich beruhten, kein Schriftzeichen für das Wort Hùng (chinesisch: hiong) sondern nur das für das Wort Lac (chinesisch: lo) feststellen. Da die beiden Schriftzeichen sehrähnlich sind, vermutet er eine Verwechselung seitens der vietnamesischen Historiographen, als sie zur Abfassung der Geschichte der Hùng-Könige die chinesischen Quellen benutzten. Nach Maspéro hat es daher keine Hùng-Könige sondern nur Lac-Könige gegeben $(\mathrm{H}$. Maspéro, Le royaume de Van-lang, in: BEFEO, XVIII, 1918, S. 7). Diese hinsichtlich der Frage der Existenz der Hùng-Könige wichtige These ist meines Wissens bisher nicht widerlegt worden. 
verdrängt, mußten sie nach Süden wandern und ließen sich schließlich im Gebiet von Tonkin etwa in der ersten Hälfte des ersten Jahrtausends v. u. Z. nieder ${ }^{11}$. Während diese These, die auf gut dokumentierten Untersuchungen fußt, vorwiegend von westlichen Historikern akzeptiert wird ${ }^{12}$, wird sie vom größten Teil der vietnamesischen Historiker zurückgewie$\operatorname{sen}^{13}$. Für sie gilt indes die Existenz vom Reich Van-lang der Hùng-Könige als unumstritten, nur hinsichtlich seiner räumlichen und zeitlichen Ausdehnung sowie seiner gesellschaftlichen und politischen Verfassung sind unterschiedliche Auffassungen vorgetragen worden ${ }^{\mathbf{1 4}}$. Besonders seit 1954 haben die Wissenschaftler der Demokratischen Republik (1945-1976) begonnen, sich systematisch mit dieser Frage zu beschäftigen, wobei versucht worden ist, zu einer einheitlichen Auffassung zu gelangen. Ergebnisse ihrer Bemühungen liegen nun in verschiedenen Veröffentlichungen vor, die in der letzten Zeit in Hanoi erschienen sind: eine umfassende vierbändige Sammlung der Diskussionsbeiträge erschien unter dem Titel „Hùng Vuong dung nuóc“ (Die Staatsgründung durch die Hùng-Könige, Bd. 1 1970, Bd. 2 1972, Bd. 3 1973, Bd. 4 1974); einen Versuch zur Darstellung der wirtschaftlichen, gesellschaftlichen, politischen und kulturellen Entwicklung des Reichs Van-lang enthält das 1971 erschienene, 1976 mit Ergänzungen neuverlegte Werk ,,Thoi dai Hùng Vuong“"(Die Epoche der Hung-Könige). Im offiziellen Geschichtswerk „,Lich su Viêt nam“ (Die Geschichte Vietnams), das 1971 von der Staatskommission für Wissenschaft (Uy ban khoa hoc Nhà nuóc) herausgegeben wurde, erreicht nun die Tendenz der Rationalisierung des Mythos von den Hùng-Königen, die bereits in den Darstellungen der früheren Hofhistoriographen feststellbar ist, ihren Höhepunkt: Das Reich Van-lang der Hùng-Könige wird endgültig als historisches Faktum behandelt.

Hier soll zunächst auf die Interpretation der vietnamesischen Historiker eingegangen werden. Ihre Darstellung beruht im wesentlichen auf der Deutung archäologischer Daten. Dazu werden auch noch Ergebnisse der Auswertung chinesischer Quellen, der vergleichenden sprachwissenschaftlichen und ethnologischen Forschung herangezogen ${ }^{\mathbf{1 5}}$.

In der Tat ist es unumstritten, daß verschiedene prähistorische Stufen auf dem heutigen Staatsgebiet Vietnams existierten. Dies wird einwandfrei bestätigt durch Ergebnisse der Ausgrabungen, die bereits zu Anfang dieses Jahrhunderts von westlichen Archäologen unternommen wurden ${ }^{16}$ und später von den Wissenschaftlern Nordvietnams intensiv weiter fortgeführt worden sind ${ }^{17}$. Die Interpretation dieser Ergebnisse ist allerdings unterschiedlich. Zum einen geht es um die Frage, ob zwischen diesen Kulturstufen, d. h. der Älteren Steinzeit und der Eisenzeit eine kontinuierliche, von endogenen Faktoren bestimmte Entwicklung stattgefunden hat. Zum anderen wird die Überlegung angestellt, ob es möglich sei, eine Verbindung zwischen den archäologischen Daten und der Uberlieferung über das Reich Van-lang der Hùng-Könige herzustellen, was naturgemäß bei den vietnamesischen Historikern großes Interesse erweckt. Während westliche Archäologen in diesen Fragen dazu neigen, die in Vietnam nachweisbaren prähistorischen Stufen als Ergebnisse eines vielfach von

11 L. Aurousseau, La première conquête chinoise des pays annamites (IIIe siècle avant notre ère), in: BEFEO, XXIII, 1923 , S. $245-264$.

12 J. Chesneaux, Geschichte Vietnams, Berlin (Ost) 1963, S. 24 f.; auch J. Villiers, Südostasien vor der Kolonialzeit (Fischer Weltgeschichte, Bd. 18), Frankfurt 1965, S. 235.

13 Lê Thanh Khoi, a. a. O., S. 22; vgl. die verschiedenen Auffassungen der vietnamesischen Historiker über diese Frage, in: Van Tân/Nguyên Linh/Lê Van Lan/Nguyên Dông Chi/Hoàng Hung, Thoi dai Hùng Vuong (Die Epoche der Hùng-Könige), Hanoi 1976, S. 19-29.

14 Van Tân, Xa hôi nuóc Van-lang và xa hôi nuoc Au-lac (Die gesellschaftliche Entwicklung von Van-lang und Au-lac), in: Nghiên cúu lich su (Zeitschrift für Geschichtsforschung NCLS), Nr. 20, 1960; Dào Duy Anh, Y kiên vê chê dô nô lê o Viêt nam (Einige Bemerkungen zur Frage der Existenz der Sklavenhaltergesellschaft in Vietnam), in: NCLS, Nr. 16, 1960; vgl. auch Nguyen Thanh Hung, Zur Rezeption der Theorie der asiatischen Produktionsweise in Vietnam, in: Osteuropa, Februar (H. 2 ) 1979.

15 Van Tân/Nguyên Linh . . ., a. a. O., S. 30-40.

16 L. Bezacier, Asie du Sud-est, tome II: Le Viêt-nam - De la préhistoire à la fin de l'occupation chinoise, Paris 1972.

17 Lê Van Lan/Pham Van Kinh/Nguyên Linh, Nhung vêt tích dâu tiên cua thòi dai dô dông thau o Viêt nam (Die ersten Spuren der Bronzezeit in Vietnam), Hanoi 1963; Etudes Vietnamiennes: Données archéologiques, Nr. 46, 1976. 
Von den vietnamesischen Vor- und Frühhistorikern periodisierte, vorgeschichtliche Zeiträume Vietnams

Steinzeit

Ältere Steinzeit

Mittelsteinzeit

Jungsteinzeit

Bronzezeit
300000 v. u. Z.

10000 v. u. Z.

3000 v. u. Z.

2000 v. u. Z.

$1000-500$ v. u. Z.

$\underline{\text { Eisenzeit }}$

\author{
Funde in Núi Do, Vietnam- \\ Mensch; Son-vì-Zivilisation \\ Hòa-binh-Zivilisation; \\ Beginn des Ackerbaus \\ Bác-son-; Bâù-tró- und \\ Ha-long-Zivilisation
}

\section{Phùng-nguyên-Zivilisation Gründungdes ReichsVan-lang durch die Hùng-Könige Dông-son-Zivilisation; Bronzetrommel con Ngoc-lu}

250 v. u. Z.

Gründung des Reichs Âu-lac; Eroberungskrieg gegen das Reich Âu-lac durch Triêu Dà

179 v. u. Z. Verfall des Reichs Âu-lac

111 v. u. Z.
Eroberung des Reichs Âu-lac durch die Chinesen (der Han-Dynastie)

äußeren Einflüssen bedingten Entwicklungsprozesses zu interpretieren ${ }^{18}$, vertreten die vietnamesischen Historiker die Ansicht, daß das Gebiet von Tonkin und Nord-Annam, also das Kerngebiet des alten vietnamesischen Staates, das Zentrum der Entstehung dieser verschiedenen Kulturen darstelle, von dem aus erst Entwicklungsimpulse nach außen gingen. Entscheindender ist dabei ihre Auffassung, daß die Träger dieser Kulturen Vorfahren der Vietnamesen seien, und sie identifizieren die Stufe der Bronzezeit mit der Epoche des Reichs Van-lang der Hùng-Könige (s. Tabelle). Diese These wird einheitlich in den erwähnten Veröffentlichungen aus Hanoi vertreten und gilt offensichtlich als offiziell anerkannt ${ }^{19}$

Das Reich Van-lang der Hanoier Historiker wurde dieser Interpretation zufolge um $2000 \mathrm{v}$. u. Z. gegründet als ein politischer Zusammenschluß verschiedener Volksstämme, die damals auf dem Gebiet von Tonkin und Nord-Annam ansässig waren. In dieser politischen Staatsgemeinschaft sollte jedoch, so wird betont, der vietnamesische Volksstamm vorherrschend gewesen sein. Bis zu seinem Untergang in den ersten Jahrhunderten v. u. Z. erlebte das Reich Van-lang eine hohe Entwicklung, deren Zeugnisse die Bronze-Zivilisation von Phùngnguyên und Dông-son seien. Die Bevölkerung lebte vornehmlich vom Ackerbau, und hier besonders vom Reisanbau ${ }^{20}$. Damit wird zugleich die These zurückgewiesen, wonach die

\footnotetext{
18 R. Heine-Geldern, Bedeutung und Herkunft der ältesten hinterindischen Metalltrommeln (Kesselgongs), in: Asia Major, vol. VIII, 1932; V. Goloubew, Sur l'origine et la diffusion des tambours métalliques, in: Praehistorica Asiae Orientalis, I, Hanoi 1932.

19 Es ist interessant festzustellen, daß Nguyên Khac Viên in seiner Veröffentlichung ,Histoire du Vietnam" (Paris 1974), die offensichtlich für das Ausland bestimmt ist, auf diese Frage nicht eingegangen ist.

20 Van Tân/Nguyên Linh . . ., a. a. O., S. 89.
} 
Reiskultur in Vietnam erst von den chinesischen Eroberern eingeführt worden sei21. Bemerkenswert ist in diesem Zusammenhang die Auffassung der vietnamesischen Historiker hinsichtlich der Interpretation der gesellschaftlichen und politischen Verfassung des Reichs Van-lang. Ging es in der Diskussion gegen Ende der fünfziger Jahre und zu Beginn der sechziger Jahre in Nordvietnam um die Frage, ob es sich hier um eine Sklavenhaltergesellschaft oder um eine Auflösungsstufe der Urgemeinschaft handele, so ist man heute der Ansicht, daß das Reich Van-lang der Hùng-Könige ,,deutliche Konturen des orientalischen Despotismus und der asiatischen Produktionsweise" aufwiese ${ }^{22}$. Anhand der dargestellten Motive auf den Funden, namentlich auf den berühmten Bronzetrommeln und der vergleichenden Untersuchungen der in den chinesischen Quellen enthaltenen Beschreibung und der Lebensweise der heutigen Bergvölker auf dem Hochland Tonkins werden dann Glauben, Sitten und Bräuche der Bevölkerung des Reichs Van-lang ermittelt ${ }^{23}$. Insgesamt läßt die Darstellung der Hanoier Historiker ein eindrucksvolles Bild von der Entwicklung Vietnams unter der Herrschaft der Hùng-Könige entstehen, die von ihnen als eine ,,glorreiche Epoche der vietnamesischen Geschichte" bezeichnet worden ist ${ }^{24}$.

$\mathrm{Zu}$ diesen Ausführungen sind allerdings einige methodische Bemerkungen notwendig. Die Interpretation der vietnamesischen Wissenschaftler beruht zunächst nicht etwa auf neuartigen Forschungsergebnissen, sondern hauptsächlich auf Daten, die bereits von westlichen Archäologen in der ersten Hälfte dieses Jahrhunderts zusammengetragen wurden. Ferner tragen die Funde keine Inschriften, denn diese Kulturen waren schriftlos. Hinzu kommt, daß die älteren schriftlichen Aufzeichnungen zu diesem Fragenkomplex auf chinesischer Seite erst aus dem dritten vorchristlichen Jahrhundert stammen. Eine derartige Materiallage beschränkt natürlich den Interpretationsrahmen erheblich. Auf jeden Fall lassen sich diese Daten nicht zu einem so vollständigen Bild der gesamten Gesellschaft zusammenfügen, wie es nun in der Darstellung der vietnamesischen Historiker vorliegt. Dies ist auch der Grund, weshalb westliche Archäologen skeptisch der Möglichkeit gegenüberstehen, die archäologischen Daten in einen Zusammenhang mit der Úberlieferung über das Reich Van-lang zu bringen ${ }^{25}$. Diese wissenschaftlich gebotene Haltung wird jedoch von den vietnamesischen Historikern als ,, kolonialistisch “ angeprangert ${ }^{26}$. Andererseits muß festgestellt werden, daß sie selbst bisher keinen wissenschaftlich überzeugenden Beweis für ihre These haben erbringen können. Die von ihnen gemachten Angaben über die räumliche und zeitliche Ausdehnung des Reichs Van-lang sind beispielsweise nicht zu überprüfen. Uberdies ist die Annahme, daß die Lebensweise der heutigen Bergvölker auf dem Hochland Tonkins noch Relikte aus der Zeit der Hùng-Könige aufweise, worauf dann ihre gesamte Darstellung der gesellschaftlichen, politischen und kulturellen Aspekte des Reichs Van-lang fußt, sehr fraglich. Angesichts dieser methodologischen Mängel, die ihnen selbst auch offenkundig sind ${ }^{27}$, ist es durchaus angebracht, Zweifel an der Richtigkeit ihrer These anzumelden. Dennoch ist ihre Interpretation nicht uninteressant, weil sie einige bemerkenswerte Züge aufweist, die über rein wissenschaftliche Probleme hinausgehen. Wie zu zeigen sein wird, entspricht die Inter-

21 J. Chesneaux, a. a. O., S. 26.

22 Pham Huy Thông, Ba lân dung nuóc (Dreimalige Gründung des Staates), in: Hoc tâp (Studien; diese theoretische Zeitschrift der Kommunistischen Partei Vietnams wurde 1977 in ,Công san“ (Kommunismus) umbenannt), 1975, H. 9, S. 9; vgl. dazu Nguyen Thanh Hung, a. a. O.

23 Van Tân/Nguyên Linh . . ., a. a. O., S. 169-233.

24 A. a. O., S. 263.

25 L. Bezacier, a. a. O., S. 14.

26 Van Tân/Nguyên Linh . . ,, a. a. O., S. 19.

27 Eine redaktionelle Bemerkung von der Zeitschrift NCLS (Nr. 97, 1967) lautet: ,,Die Schlußfolgerungen unserer Historiker hinsichtlich der Frage der Hùng-Könige sind wissenschaftlich und ideologisch zwar sehr wertvoll, können bisher jedoch nicht alle überzeugen, wie wir dies gewünscht hätten". 
pretation der vietnamesischen Historiker eher den aktuellen politisch-ideologischen Geboten als den wissenschaftlichen Bedürfnissen, wobei ihre Intention sich kaum von derjenigen der Urheber des Mythos von den Hùng-Königen im 14. Jahrhundert unterscheiden dürfte. Es liegt durchaus nahe, anzunehmen, daß die Hanoier Wissenschaftler mit der Konstruktion einer blühenden Epoche der Hùng-Könige in der vietnamesischen Geschichte bewußt einen neuen Mythos schaffen wollen.

Zunächst soll angemerkt werden, daß die Forschung über das Reich Van-lang der HùngKönige zwar seit 1954 in Nordvietnam im Gange ist, jedoch gegen Ende der sechziger Jahre besonders intensiv betrieben wurde. An einem dreijährigen Forschungsprojekt über die „,Epoche der Hùng-Könige“ (1968-1971) nahmen fast alle Historiker Nordvietnams teil28. Das große Interesse für eine so weit zurückliegende geschichtliche Epoche, das darüber hinaus gerade in der härtesten Phase des Vietnam-Krieges auftauchte, wird kaum verständlich, wenn ihre aktuelle Bedeutung nicht voll erkannt wird, und sie wird bezeichnenderweise von dem Ministerpräsident Nordvietnams Pham Van Dông so formuliert:

„,Dieses Problem steht in engem Zusammenhang mit der Erforschung des Ursprungs unseres Volkes, unserer Gesellschaft, und somit auch unmittelbar mit den Ereignissen unserer Geschichte in den letzten Jahrzehnten, also mit wichtigen Fragen unserer Generation, ja sogar der nächsten Generation unseres Volkes . . . Wir stehen hier also vor einem großen, neuen Problem, das sich nicht nur auf das vietnamesische Volk, sondern auch auf andere Völker in Südostasien bezieht. Aus diesen Gründen denke ich, daß eine ganz besonders große Bedeutung der Erforschung dieser Epoche zukommt, deren Ergebnisse niemand von uns jetzt schon zu würdigen vermag. ${ }^{\prime 29}$

Es ist also kaum zu übersehen, daß die Forschung über die Epoche der Hùng-Könige nicht nur von historischem Interesse, sondern auch durch politisch-ideologische Faktoren bedingt ist. Angesichts dieser Formulierung von einer politischen Autorität wie Pham Van Dông muß sogar angenommen werden, daß das wissenschaftliche Interesse dabei nur eine vordergründige Rolle spielt. In diesem Zusammenhang erscheint die Interpretation der Epoche der Hùng-Könige von den vietnamesischen Historikern in der Tat für unser Verständnis der politisch-ideologischen Orientierung der vietnamesischen Führungsgruppe als sehr aufschlußreich, denn im neuen Mythos von den Hùng-Königen kann sich gerade das nationale Selbstverständnis der Vietnamesen unverhüllt artikulieren, das sich sonst in der euphemistischen Sprache der offiziellmarxistischen Ideologie schwer erkennen läßt. Einige bedeutsame Aspekte dieser Erscheinung sollen im folgenden aufgezeigt werden.

Wir haben bereits erwähnt, daß die Nachbarschaft einer mächtigen alten Kultur im Norden frühzeitig in Vietnam das Bedürfnis entstehen ließ, die eigene Kultur als ebenbürtig darzustellen. Dieses Bedürfnis ist heute in Vietnam trotz der neuen historischen Konstellation immer noch vorhanden. In der jetzigen Version über das Reich Van-lang reduziert man zwar die in der ursprünglichen Fassung aus dem 14. Jahrhundert angegebene beträchtliche territoriale Ausdehnung auf eine realistischer erscheinende Größe, behält jedoch das willkürlich angegebene hohe Alter bei. Während die früheren Hofhistoriographen sich in ihrer Darstellung ausschließlich an den Maßstäben der chinesischen Welt orientierten, ist der Bezugsrahmen der heutigen Interpretation größer geworden. Das Reich Van-lang der Hanoier Historiker läßt sich nicht nur in jeder Hinsicht mit dem alten China messen, sondern Vietnam

28 Pham Huy Thông, Báo cáo chung tai hôi nghi khao cô hoc bàn viêc nghiên cúu thòi kỳ lich su Hùng Vuong (Allgemeiner Bericht an die archäologische Tagung über die Forschung der Epoche der Hùng-Könige), in: Hùng Vuong dung nuoc (Die Staatsgründung durch die Hùng-Könige), in: Bd. 1, Hanoi 1970, S. 21

29 Pham Van Dông, Bài nói tai hôi nghi khao cô hoc bàn vê viêc nghiên cúu thòi kỳ lich su Hùng Vuong (Rede an der archäologischen Tagung über die Forschung der Epoche der Hùng-Könige), in: Hùng Vuong dung nuoc, a. a. O., S. $7 \mathrm{f}$. 
stellt sich nach ihrer Auffassurig auch als eine der Urheimaten der Menschheit überhaupt $\mathrm{dar}^{30}$. Die Epoche der Hùng-Könige wird ferner von ihnen als eine der am höchsten entwikkelten Bronzekulturen der Welt charakterisiert. Um dieser Intention gerecht zu werden, scheuen die vietnamesischen Wissenschaftler sich nicht, sich auch sehr fragwürdiger Deutungsmethoden zu bedienen. Ein typisches Beispiel dafür ist ihre Interpretation des berühmten Motivs auf der Bronzetrommel von Ngoc-lu. Auf dieser Trommel ist ein Schiff zu erkennen, dessen Besatzung am Kopf Federschmuck trägt. Dieses Motiv, das offensichtlich eine kultische Bedeutung hat, wird von ihnen ,,materialistisch“ dahingehend gedeutet, daß seine Schöpfer genau gewußt hätten, daß die Bronzetrommel übers Meer zu ihrem Bestimmungsort gebracht werden sollten ${ }^{31}$ ! Damit soll nämlich ihre These gestützt werden, daß Vietnam schon sehr früh seine Produkte in die fernen Länder exportiert hätte. Besonders fragwürdig ist die Behauptung, daß der vietnamesische Volksstamm zur Zeit des Reichs Van-lang dank seiner ,,Lebenskraft" aus allen anderen Volksstämmen in Südostasien herausragen sollte ${ }^{32}$. Um diese Behauptung zu stützen, machen sich die Hanoier Historiker selbst jene berüchtigte sozialdarwinistische Auffassung zu eigen, wonach nur ein zahlenmäßig großes, von Lebenskraft erfülltes Volk sich in der Geschichte der Menschheit durchsetzen könne ${ }^{33}$. Und zu diesen , lebenskräftigen“ Völkern zählen sie selbstverständlich den vietnamesischen Volksstamm.

Somit impliziert derneue Mythos von den Hùng-Königen den Anspruch, daß die Vietnamesen aufgrund ihrer historischen, kulturellen Größe und ihrer ethnischen Beschaffenheit eine angemessene Stellung in der heutigen Welt haben müßten. Darüber hinaus ist die Darstellung der Epoche der Hùng-Könige besonders gekennzeichnet durch die Bemühungen der Historiker, die damaligen sozialen und politischen Verhältnisse zu idealisieren. So sollte sich bei dem Reich Van-lang zwar um einen Vielvölkerstaat unter der Dominanz des vietnamesischen Volksstammes handeln, doch sollen all diese Volksstämme nur friedlich nebeneinander gelebt haben ${ }^{34}$, ebenso sollen die Beziehungen zwischen Herrschenden und Beherrschten frei von Konfliktengewesen sein. Die Bevölkerung habe hauptsächlich aus Ackerbauern bestanden, die sich in auf dem Gemeineigentum an Grund und Boden beruhenden Dorfgemeinschaften organisierten ${ }^{35}$. Die Herrschenden sollten sich übrigens auch noch nicht von der unmittelbaren materiellen Produktion losgelöst haben und gegenüber ihren Untertanen sehr fürsorglich gewesen $\operatorname{sein}^{36}$. Um diese soziale und politische Eintracht marxistisch zu deuten, bedienen sich die vietnamesischen Historiker statt des sonst obligatorischen vom Klassen antagonismus beladenen Konzeptes der Sklavenhaltergesellschaft der Kategorie der asiatischen Produktionsweise, deren ursprünglich von K. Marx gegebener kritischer Gehalt von ihnen freilich ignoriert wird. Das so idyllisch anmutende Reich Van-lang wurde jedoch durch den Einfall der Fremden aus dem Norden im dritten Jahrhundert v. u. Z. zerstört. Hier wird dann die ideologische Implikation des neuen Mythos von den Hùng-Königen

30 Lich su Viêt nam (Geschichte Vietnams), Hanoi 1971, S. 31.

31 Van Tân/Nguyên Linh . . ., a. a. O., S. 114

32 A. a. O., S. 141.

33 Interessanterweise berufen sich die vietnamesischen Historiker a n dieser Stelle auf den ,Klassiker“ F. Engels. So schreiben sie wörtlich: ,,Engels hat die Völker der Welt in zwei Arten aufgeteilt: Die eine ist zahlenmäßig groß, hat eine große, stabile nationale Lebenskraft; die andere ist zahlenmäßig klein, hat eine schwache Lebenskraft und diese Völker sind leicht auflösbar, ,(a. a. O., S. 139). Diese Autoren verweisen dabei auf eine von Engels im Zusammenhang mit der Frage der Nationalität gemachte Äußerung, die jedoch so lautet: , Das Nationalitätsprinzip wirft zwei Arten von Fragen auf: erstens Fragen nach den Grenzen zwischen diesen großen historischen Völkern und zweitens Fragen des Rechts der zahlreichen kleinen Uberbleibsel jener Völker auf unabhängige nationale Existenz, die, nachdem sie längere oder kürzere Zeit auf dem Schauplatz der Geschichte aufgetreten sind, schließlich als Bestandteile in diese oder jene mächtigere Nation eingingen, welche vermöge ihrer größeren Lebenskraft imstande war, größere Hindernisse zu überwinden" (F. Engels, Was hat die A.rbeiterklasse mit Polen zu tun? in: Marx Engels Werke, Bd. 16, Berlin 1973, S. 158).

34 Van Tân/Nguyên Linh . . ., a. a. O., S. 254

35 A. a. O., S. 147.

36 A. a. O., S. 145. 
deutlich: Durch die fremden Eindringlinge begann erst das Leiden des vietnamesischen Volkes. Der ,Sündefall“ in der vietnamesischen Geschichte ereignete sich nicht, wie es in der marxistischen Tradition der Historiographie üblich ist, durch die innere, aufgrund der Entstehung des Privateigentums bedingte Spaltung der Gesellschaft in soziale Klassen ${ }^{37}$, sondern eben durch eine fremde Eroberung. In diesem Kontext erscheint die vietnamesische Geschichte als ein ununterbrochener Kampf gegen die Ursache dieses Leidens. Zu dieser Tradition gehört selbstredend der Krieg gegen die Legitimität der Führung der kommunistischen Partei begründet liegen, die aus diesem Verständnis nichts anderes als das ursprüngliche Glück der Vietnamesen wiederherzustellen versuchte.

Der neue Mythos von den Hùng-Königen spiegelt auch einen anderen ideologischen Konflikt wider. Es ist durchaus kein Zufall, daß gerade gegen Ende der sechziger Jahre die idealisierte Deutung der Epoche der Hùng-Könige von den vietnamesischen Historikern herausgestellt wurde. Sie fiel mit der feststellbaren ideologischen Desorientierung der Parteiführung angesichts der offenkundig gewordenen Aussichtslosigkeit einer Beilegung des sinosowjetischen Konflikts zusammen ${ }^{38}$. Wenn Hô Chí Minh in den fünfziger Jahren noch so unbekümmert aussprechen konnte, daß ,,das Heute der Sowjetunion und der Volksrepublik China das Morgen Vietnams" sei, so war eine derartige Identifikation gegen Ende der sechziger Jahre aufgrund der Entwicklung in diesen beiden ,,Großbruderländern“ überhaupt nicht mehr denkbar. Der einst so unerschütterlich scheinende Glaube an die Verwirklichung der idealen Gesellschaft, an die ,,große sozialistische Gemeinschaft" wurde plötzlich durch dieses Ereignis in Frage gestellt. Diese den Vietnamesen als widrig erscheinende realpolitische Tatsache wurde dann mit der Idealisierung ihrer eigenen Vergangenheit überspielt, woraus sie zugleich neue Kraft für ihren Glauben zu schöpfen suchten. So begann man gegen Ende der sechziger Jahre sich auf einen ,,nationalen Weg zum Sozialismus“ zu besinnen ${ }^{39}$. Gesucht und auch entdeckt wurden dabei allerlei „Nationalcharakter" (Tinh cách dân tôc) wie Arbeitssamkeit, Solidarität, ausgeprägte kollektive Verhaltensweise . .., also durchweg Eigenschaften, die im Grunde genommen in jeder vorindustriellen Gesellschaft nachgewiesen werden können. Dieser Selbstfindungsprozeß gipfelte dann in der Konstruktion des Mythos von einer Epoche der Hùng-Könige, die die Verwirklichung aller zu erstrebenden menschlichen Werte scheinbar antizipiert und offensichtlich die Projektion der eigenen kommunistischen Zukunft ist. Heute spricht man in Vietnam selbstbewußt von einem ,,vietnamesischen Weg zum Sozialismus“40 was bis zur Mitte der sechziger Jahre noch undenkbar gewesen wäre. So bedeutet der neue Mythos von den Hùng-Königen den Vietnamesen nicht nur eine ideologische Zuflucht in der sino-sowjetischen Auseinandersetzung sondern auch eine ideologische Neuorientierung.

In engem Zusammenhang damit stellt die Idealisierung der Epoche der Hùng-Könige eine Fetischierung von Volk und Nation dar, von Begriffen also, die sonst in der marxistischen Theorie bestenfalls als historische Kategorien auftreten. Es ist nicht uninteressant hier auf die Fragestellung der vietnamesischen Historiker bezüglich der Entstehung der vietnamesischen Nation in der Diskussion der fünfziger Jahre zurückzukommen. Drei Thesen wurden damals in der Auseinandersetzung vorgetragen: Die Herausbildung der vietnamesischen $\mathrm{Na}-$ tion wurde entweder mit der Entstehung des ,,feudalen Staates“ (im 11. Jahrhundert), oder mit dem Verfall des ,, vietnamesischen Feudalismus“ (gegen Ende des achtzehnten Jahrhun-

\footnotetext{
37 F. Engels, Der Ursprung der Familie, des Privateigentums und des Staates, Berlin 1946.

38 Vgl. Nguyen Thanh Hung, Grundzüge des ideologischen Bezugsrahmens der Kommunistischen Partei Vietnams, vorgetragen an der Vietnam-Tagung in Hamburg im Oktober 1978.

39 A. a. O.

40 Duòng lôi cách mang Viêt nam (Richtlinien der vietnamesischen Revolution), Hanoi 1977.
} 
derts) oder mit der Herausbildung der Arbeiterklasse und der Entstehung der kommunistischen Bewegung (in den zwanziger Jahren dieses Jahrhunderts) identifiziert ${ }^{\mathbf{4 1}}$. Diese Thesen sind zwar in vieler Hinsicht sehr problematisch, weisen jedoch gemeinsam auf den historischen Charakter der vietnamesischen Nation auf. Von diesen Auffassungen scheinen sich die vietnamesischen Historiker heute zu entfernen, da der neue Mythos von den Hùng-Königen die Geburt der vietnamesischen Nation mit dem Beginne der vietnamesischen Geschichte gleichsetzt. So schreiben die Autoren des neuen Mythos:

,,Während der Entwicklung in der Epoche der Hùng-Könige läßt sich bereits die Herausbildung stabiler Konstituenten der vietnamesischen Nation feststellen, wodurch sich die Vietnamesen von anderen Völkern unterschieden. Der Unterschied bestand nicht nur im Bereich der Sprache sondern hauptsächlich auch in anderen Aspekten ihrer Lebensweise.

Als die Vietnamesen im Delta des Roten Flusses seßhaft wurden, hatten sie bereits ihre eigenen Sitten und Bräuche: Sie färbten ihre Zähne, kauten Betel, tätowierten ihren Körper nach dem Bild der Krokodile. Sie pflegten den Ahnenkult, verehrten ihre Nationalheroen. Ihr Lebensverständnis war von einer kollektiven Einstellung, vom Geist der Solidarität, von einem vom Pragmatismus geprägten Denken und Handeln bestimmt. Sie waren stolz auf ihre Abstammung von den Geschlechtern des Drachens und der Fee usf.

All dies drang während jener Zeit immer tiefer in das Bewußtsein der Vietnamesen und bildete die Grundlage ihres Nationalcharakters . . .

Dieses Nationalbewußtsein wurde dann die Quelle jener Kraft, mit der die Vietnamesen ihren Staat in der Epoche der Hùng-Könige gründeten und verteidigten, mit der sie sich später während der chinesischen Herrschaft behaupteten und nach dem Erlangen der Unabhängigkeit weiter entfalteten." 42

Das vietnamesische Volk und die vietnamesische Nation, was sich übrigens in der heutigen Sprache in Vietnam in einem Wort (dân tôc) ausdrücken läßt, hätten nach dieser Auffassung nicht etwa im geschichtlichen Prozeß erst an Konsistenz gewonnen, sondern sind durch diesen Prozeß hindurch vielmehr unverändert geblieben. Die Auffassung, daß die Herausbildung der vietnamesischen Nation nicht etwa als Ergebnis einer politischen, sozialen und kulturellen Emanzipationsbewegung zu begreifen ist, sondern vielmehr aus den ,,Wesenszügen" der Vietnamesen selbst hervorgegangen sein sollte, läßt die Idee des Nationalismus in Vietnam durchaus als anachronistisch erscheinen. Volk und Nation werden so etwas wie eine Entität aufgefaßt und bestimmen als solche die politische Kultur sowohl der Elite als auch der Masse. Es bestehen kaum Zweifel, daß gerade die anachronistischen Züge des vietnamesischen Nationalismus eine wichtige Funktion in der Mobilisierung der Bevölkerung im Kampf gegen die Franzosen und Amerikaner erfüllt hat, doch künftig auch als Hindernisse für eine rationale Gestaltung der Politik Vietnams darstellen könnten.

Seit dem Ausbruch der offenen Auseinandersetzung mit den Nachbarländern - Seit Dezember 1977 mit Kampuchea und seit Mai 1978 mit China - scheint der neue Mythos von den Hùng-Königen wieder an aktueller Bedeutung gewonnen zu haben. Im Hinblick auf diese Ereignisse wird in der theoretischen Zeitschrift der Kommunistischen Partei Vietnams „Công san“ (Kommunismus) nachdrücklich betont, daß bereits seit der Zeit der Hùng-Könige in Vietnam und in China zwei unterschiedliche Kulturen entstanden seien, daß ihre Beziehungen zueinander sich nur in der Form eines Süd-Nord-Dialogs abgespielt hätten ${ }^{\mathbf{4 3}}$. Abermals muß die Existenz der Hùng-Könige herausgestellt werden:

\footnotetext{
41 Phan Gia Bên, La Recherche historique en République Démocratique du Vietnam 1953-1963, Hanoi 1965 , S. 64 f. 42 Van Tân/Nguyên Linh . . ., a. a. O., S. 261.

43 Trân Quôc Vuong, Tù thòi dai Vua Hùng dên thòi dai Hô Cií Minh (Von der Epoche der Hùng-Könige zur Epoche von Hô Chi Minh), in: Công san (Kommunismus), 1978 H. 9, S. S. 16 f.
} 
„,Das Reich Van-lang der Vietnamesen und der Hùng-Könige bestand gleichzeitig mit den drei Ältesten Dynastien Chinas (Hsia, Shang und Chou). Dies ist ein historisches Faktum und nicht ein Produkt der Phantasie der vietnamesischen Historiker; die Geschichte Vietnams beginnt nicht erst seit dem X. Jahrhundert n. u. Z., sogar viel später als die von Kampuchea, wie dies neulich in einer chinesischen Zeitschrift grob und in heimtückischer Absicht behauptet worden ist. " 44

Konnte bisher der Mythos von den Hùng-Königen Vietnam als Opfer der Fremdherrschaft darstellen und so eine mobilisierende Funktion haben, so scheint seine neue Version heute da Vietnam inzwischen selbst eine Großmacht geworden ist - dafür geeignet zu sein, zur Legitimierung einer Politik zu dienen, die den eigenen nationalen Standpunkt verabsolutiert. Angesichts der Tatsache, daß Vietnam seit 1975 bestrebt ist, eine dominierende Stellung in Indochina und Südostasien einzunehmen und im Zug dieser expansionistischen Politik eigene Truppen in Laos und Kampuchea unterhält, ist die Frage durchaus angebracht, ob die politische Führung Vietnams bereits Gefangene ihres Mythos geworden ist.

44 A. a. O., S. 19. 


\title{
Legalism Rampant: The Heritage of Imposed Law and the Constitution of Papua New Guinea.
}

\author{
By JOHN GOLDRING
}

The Constitution of Papua New Guinea, which came into force when Papua New Guinea became Independent, is autochthonous. It seeks to give, of its own force, legal effect to all the statute and judge-made law of the country, so that the authority of law is in no way dependent on any colonial law in force prior to Independence. It also seeks to establish the customs of the people, which were scarcaly recognised by the legal system prior to Independence, the status of ,underlying law' and to require the legislature and the courts to give effect to the traditions of the people. The Constitution itself calls for great significance to be attached to ,Papua New Guinean Ways”. The Constitution is the result of a process of popular consultation unparallelled in any other country.

Despite this, the Constitution of Papua New Guinea is firmly rooted in the traditions of Western culture, specifically, the Westminster system of government and the English Common Law. It is a complicated and highly legalistic document, which depends for its enforcement on judicial review and enforcement by a court and lawyers trained in the Common Law and brought up to respect the traditions of responsible and representative government. Despite the exhortations in the Constitution to respect and enforce the traditions of the country, it is itself an instrument which requires for its effectiveness a legal culture which is not indigenous to Papua New Guinea, but is, in fact, the heritage of the Common Law which was imposed upon the country by its colonial rulers.

\section{Labour Legislation in Algeria}

\section{By GUNTER WIEDENSOHLER}

The Algerian Law No. 78-12 of 5th August, 1978 (Statut général des travailleurs) is a significant example for the concept of labour law in a socialist country in development. A remarquably high importance is given to labour relations even in the Charter and the Constitution. Further it is surprising to see that freedom to strike does not exist in the public economic sector nor the principle of state sovereignty of collective bargaining, social security regulations. At various places there are hints to the social obligation to work, the principle of achievement motivated remuneration and to the significance of the vocational school system. The new law is expected to lead to a higher standard of discipline and responsibility within the labour relations.

\section{The Myth of the Hung-Kings and the National Self-Evidence of the Vietnamese}

The fact that marxism is the ruling ideology in Vietnam of ten seems to have the appearence to have overcome nationalism, which, otherwise, represents the most effective political ideology in the Third World. It is the task of this essay to prove by means of an analysis of the official interpretation on the myth of the Hung-kings that this fact is deceptive. These Hungkings were the legendary founders of the Vietnam state about whom there has been much report in the vietnamese annales. In the quest for nationalidentity the historians of the Socialist 
Republic of Vietnam have attempted by means of a dubious interpretation to represent not only the legendary dynasty of the Hung-kings as a historical phenomenon as well as the golden age in vietnamese history, but also to emphasize the cultural and ethnic superiority of the Vietnamese as a opposed to other neighbouring nations. This interpretation causes the more than 1000 years chinese leadership as well as the french colonial leadership and the subsequent cultural development of Vietnam to appear episodical and marginal. There is no doubt that this interpretation was of the utmost significance for forming a national conscience during the liberation phase under french colonialism. It gives cause to think that particularly during the present time in Vietnam this interpretation is given even more attention, especially in the view of the fact that Vietnam itself has become a regional super power since 1975.

\section{Süd/Süd-Beziehungen und die Veränderung der Position der Peripherien in der Welt- gesellschaft (Inter-periphery relations as a factor modifying the position of peripherical societies within the world society)}

\section{By BASSAM TiBI}

A currently debated question is whether underdevelopment can best be overcome by a strategy of dessociation from the world market or via greater integration of the underdeveloped countries (peripheries) in world trade and the world market (New International Economic Order). The author argues that this debate is being carried on in blissful ignorance of classical epistemological theory in that general concepts are employed devoid of any corresponding empirical content and thus fail to mediate between the particular and the general in the Hegelian sense. General concepts in which the particular is not subsumed are empty notions. The author therefore pleads for an approach to development strategy based first on empirical findings which might then serve as a guide in conceptualization and the development of a general theory. He attempts to illustrate this in his treatment of OPEC strategy in the world society.

Inter-periphery relations were formerly a matter of rather loose, non-specific, and heterogeneous political associations, such as the non-aligned, Afro-Asian movement, incapable of bringing about any changes. The OPEC in contrast is a trade union - like interest association which has had a major impact. Prior to its foundation multinational companies were able to manipulate posted prices at will. First the OPEC achieved stabilization of oil prices and hence stabilization of members' income from oil. Later they were able to put through massive price increases. Currently the OPEC is striving to increase participation in decision-making processes hitherto determined by the oil companies. The OPEC example shows how a quasi trade union type of organisation among underdeveloped countries having homogeneous and specific interests in common is capable of altering structures of the world society in favour of the periphery. Whether or not this hypothesis may be generalized can only be decided by means of further empirical research; it can be neither confirmed nor disproved by purely conceptual argument. The question of underdeveloped countries' increased revenue from raw materials exports must, however, be dealt with in connection with the question of what is done with this income. A trade union-like strategy ought only to be promoted when such revenues are used to finance the overcoming of underdevelopment. 